Bull. Fac. Agric., Cairo Univ., 71: 243-250 (2020).

\title{
USERS' OPINION ON THE MOBILE APPLICATIONS FOR THE INTEGRATED MANAGEMENT OF POMEGRANATE AND OLIVE FRUIT CROPS
}

(Received: 25.11.2020)

\author{
By \\ A.A. Farag, A.A. El-Taweel*and Engy Kh. Faied** \\ Center Laboratory for Agricultural Climate, *Horticultur Research Institute, and \\ **Agricultural Extension \& Rural Development Research Institute, \\ Agricultural Research Center, Giza, Egypt
}

\begin{abstract}
A survey was designed and administered on a sample of fruit growers to identify their perceptions and experience with the newly developed E-extension mobile application for Pomegranate and Olive. This was achieved through investigating a number of related sub-objectives covering some dimensions of the mobile application including: 1) Identify the opinion of the respondents on the degree of easiness in using the mobile application as an extension method. 2) Identify the opinion of respondents regarding the quality of the agricultural information loaded on the applications. 3) Identify the opinion of the respondents regarding the quality of the pictures and videos of the applications. 4) Identify the respondents' willingness to recommend the mobile applications to others. 5) Identify the users' suggestions of other crops that need to develop mobile applications for them. The data were collected from users of the integrated management mobile applications for Pomegranate and Olive. Two simple random samples were drawn using the Krejcie \& Morgan formula. The sizes of the samples were 227 users of the Pomegranate mobile application, and 239 users of the Olive mobile application respectively. Data were collected through personal interview using a pretested questionnaire during September 2020. Percentages, frequencies, mean and standard deviation, were used for data presentation in the findings. The main results showed that, regarding the opinion of the respondents on the quality of the pictures and videos of the applications, the majority of the Pomegranate application users $72.2 \%$ are concentrated in the high rating category, against $61.5 \%$ of the Olive application users. Regarding the respondents' willingness to recommend the mobile applications to others, it was found that the majority of the Pomegranate application users $(82.8 \%)$ in the high rating category. Also, according to the questioner, Watermelon, then Mango and Guava were selected by respondent to apply within E-Extension. These results indicated that the mentioned applications were useful for their users.
\end{abstract}

Key words: digital agriculture, E-extension services, mobile applications, Pomegranate, Olive

\section{INTRODUCTION}

The rise of digital agriculture could be the most transformative and disruptive of all the industries, because digital agriculture not only will change how farmers farm their farms, but also will transform fundamentally every part of the agrifood value chain. Digital agriculture will affect the behavior of farmers, and also affect the way that input providers, processing and retail companies market, price and sell their products, (Trendov, et al., 2019).

Agricultural extension is a key factor in agricultural development and it is the system responsible for transfer the applicable researches to the beneficiaries in farms and rural communities, aiming to increase agricultural production, the national income, and the income of the rural families.

E-extension services-as a fast growing feature of E-agriculture can help raise farm profits and provide a cost-effective way to reach a greater number of farmers. They can provide farmers with relevant and real-time access to information on how to resolve both general and specific problems, ranging from sustainable farming practices, climate-smart solutions, and market access. Extension agents can use a 
combination of digital technologies to reduce the cost and increase the frequency of interaction with farmers. (Townsend et al., 2019).

Mobile communications technology has quickly become the world's most common approach of transmitting voice, data, and services in the developing world. Given this dramatic change, mobile applications (m-apps) in general and mobile applications for agricultural, and rural development (m-ARD apps) in particular, hold significant potential for advancing development. They provide the most affordable means for millions of people to access information, markets, finance, and governance systems which were previously inaccessible. The information that this service provides includes appropriate agricultural innovations and practices, as well as weather news (Qiang, et al., 2011).

The mobile app is one of the platforms, where a farmer can get all solutions and information by just one touch. Smartphone apps revolutionized the connectivity and used for transferring agri-information to farmers. According to the statistics, there are two billion smartphones user across the globe in 2016 (EMarketer. 2016).

Simple mobile phones can be used as a means of collecting both farmers and agentlevel data, thereby improving the accountability of extension services. Voice calls and SMS between farmers and extension agents can be used to collect data on the use of new technologies, costs and yields on a more frequent basis, rather than waiting for annual agricultural surveys, when recall data on costs and production are often subject to measurement error. In addition, mobile phones can be used to verify agents' visits, as has been done with cameras in schools in India (Duflo et al., 2007). Both of these applications could improve the monitoring of extension systems (Aker, 2010).

It is important to assess the opinions of the users' of the agricultural applications in order to help optimizing the benefits of these applications. The assessment would cover identifying the perceptions of the users with regards to their experience with using the applications for Pomegranate and Olive as Eextension services.

\section{Previous studies in Egypt}

The Egyptian Ministry of Agriculture has launched, the mobile extension initiative through cooperation between the Central Administration of Agricultural Extension Service (CAAES), the Agricultural Research Center and Vodafone Telecom, in 2011, through a working protocol and coordination with Quick Surf to obtain the database and receive information from the Central Administration for Agricultural Extension and send it to the farmers. (Kassem and Shabana. 2017)

A study to assess the efficiency of mobile application on Olive was conducted in Ismailia. The study conducted a survey on 291 farmers and found that $22 \%$ of the respondents indicated that the efficiency of the extension was low or inactive in the application of technical recommendations for the integrated control of the Olive fruit fly \& $62 \%$ said that its efficiency was moderate, while only $6 \%$ mentioned that its efficiency was high. (Mohamed et al., 2018).

In another scientific attempt to explore the challenges facing farmers in benefiting from extension services provided through mobile phones. A study was carried out in Dakahalia governorates. The data were collected by a questionnaire through personal interview during the period from January to March 2016. The main findings of the study recorded the main challenges facing farmers in utilizing mobile phone in accessing the extension services were: Lack of awareness among farmers about the benefits of mobile- extension (89.02\%), and prevailing the illiteracy among farmers (75.4\%). (Abd El-Magieed et al.,2016).

\section{The problem of the research:}

It is very important to assess the agricultural mobile applications by conducting Users' Opinion survey for optimizing the benefits of these applications. What is the perception of the users with regards to their experience while using the applications for Pomegranate and Olive as an E-extension services? This main question was presented as the main problem of the research which can be answered by answering the following research questions:

1) What is the opinion of the respondents on the degree of easiness in using the mobile application as an extension method?. 2) What is the opinion of respondents regarding the quality of the agricultural information loaded on the applications?. 3) What is the opinion of the respondents regarding the quality of the pictures and videos of the applications? 4) What is the respondents' willingness to recommend the mobile applications to others?. 5) What is the 
users' suggestions on other crops that need to develop mobile applications for them?

\section{Objectives}

This study aims to identify the perceptions and experience of the respondents with the newly developed E-extension mobile application for Pomegranate and Olive. This objective was achieved through investigating a number of related sub-objectives covering some dimensions of the mobile application including:

1) Identify the opinion of the respondents on the degree of easiness in using the mobile application as an extension method.

2) Identify the opinion of respondents regarding the quality of the agricultural information loaded on the applications.

3) Identify the opinion of the respondents regarding the quality of the pictures and videos of the applications.

4) Identify the respondents' willingness to recommend the mobile applications to others.

5) Identify the users' suggestions on other crops that need to develop mobile applications for them.

\section{METHODOLOGY}

Integrated olive and pomegranate mobile applications are mobile applications which are related to olive and pomegranate to provide information about cultivation, varieties, irrigation, fertilizer, pest and disease and production. All information and equation for this application were collected from private farms and ARC researchers and agricultural experts for Olive and Pomegranate. It provides information for farmers as a package of integrated crop production. This app is developed by Central Laboratory for Agricultural Climate (CLAC) Agriculture Research Center (ARC). Digital technology and agricultural expertise and knowledge have been merged, thus an assortment of smartphone apps according to the needs of farmers has been evolved. The development of the interactive application environment of this app mobile is based mainly upon the use of object-oriented and visual development tools. Android studio mobile Platform gives everything needed to build Android applications on mobile application. The Integrated crop management system is built using object-oriented visual languages (an integration of web development languages
HTML, Java script and Cascading Style Sheets and java).

A sample survey was designed and administered on a sample of fruit growers to identify their perceptions and experience with the newly developed E-extension mobile application for pomegranate and olive.

The data were collected from two samples drawn from two populations, the first consists of 555 users of the integrated management mobile applications for Pomegranate and the second consists of 633 users of the integrated management mobile applications for Olive. Two simple random samples were drawn according to the Krejcie and Morgan equation (1970).

\section{$\mathbf{S}=\mathrm{X}^{2} \mathbf{N P}(\mathbf{1 - P}) / \mathrm{d}^{2}(\mathbf{N}-1)+\mathrm{X}^{2} \mathrm{P}(\mathbf{1 - P})$}

Where: $S=$ Required Sample size $X=Z$ value (e.g. 1.96 for $95 \%$ confidence level) $\mathrm{N}=$ Population Size $\mathrm{P}=$ Population proportion (expressed as decimal) (assumed to be $0.5(50 \%)$ $\mathrm{d}=$ Degree of accuracy (5\%), expressed as a proportion (.05).

The size of the samples was 227 and 239 for the users of pomegranate and olive applications respectively. Data were collected using a pretested questionnaire through personal interview during September 2020. For data analyzing, percentages and frequencies, mean and standard deviation were used.

\subsection{Operational definition and Measurement of variables}

2.1.1. Opinion of the respondents on the easiness in using the applications as an extension method:

This variable was defined as the total number of the respondents approval ratings for 15 statements dealing with the following points: The easiness in installing the application on the mobile, the clarity of the application menu options, the easiness of dealing with options and the appropriateness of the application area, the need to develop the application, the absence of the need to explain the application, appropriateness of the application colors to eye vision and the adoption of many farmers to use the applications.

The responses were classified to (agree, agree to some extent, disagree) for each statement separately, and the scores were given $3,2 \& 1$ or vice versa for each arrangement according to the negativity or positivity of the statement. 


\subsubsection{The opinion of respondents regarding the quality of the agricultural information loaded on the applications}

This variable was defined as the total number of the respondents 'agreement ratings for 9 statements dealing with the degree of accuracy, clarity, applicability, and ease of written language of agricultural information provided through the applications.

The responses were classified to (agree, agree to some extent, disagree) for each statement separately, and the scores were given $3,2 \& 1$ or vice versa for each arrangement according to the negativity or positivity of the statement.

2.1.3. The opinion of the respondents regarding the quality of the pictures and videos of the applications

This variable was defined as the total number of the respondents 'agreement ratings for 4 statements dealing with the opinions of users of applications regarding the clarity of images and videos, the clarity of the sound of the videos, and their preference for using pictures and videos.

The responses were classified to (agree, agree to some extent, disagree) for each statement separately, and the scores were given $3,2 \& 1$ or vice versa for each arrangement according to the negativity or positivity of the statement.

2.1.4. The respondents' willingness to recommend the mobile applications to others:

This variable was defined as the total number of the respondents agreement ratings for 2 statements dealing with the respondents' willingness to recommend the mobile applications to others. The respondents' responses were classified to (agree, agree to some extent, disagree) for each statement separately, and the scores were given $3,2 \& 1$ or vice versa for each arrangement according to the negativity or positivity of the statement.

\section{RESULTS AND DISCUSSION \\ 3.1. Respondent's opinion on the easiness of using the applications as an extension method:}

The results in Table (1) show that the theoretical range for this variable ranged from a minimum of 15 degrees to a maximum of 45 degrees, while the actual range falls between a minimum of 21 degrees to a maximum of 39 degrees for the ratings of the Pomegranate mobile application users, with a mean of 31.65 degrees, and a standard deviation of 3.98. The results in Table (2) regarding the Olive mobile application show that the actual range falls between a minimum of 22 degrees to a maximum of 44 degrees, for the ratings of the olive mobile application users, with a mean of 31.18 degrees and a standard deviation of 3.66.

The theoretical range of the measures of the studied variable was used to construct three categories, 1) a low rating category that ranged from15 degrees to less than 25 degrees, 2) a medium rating category that ranged from 25 degrees to less than 35 degrees, and 3) a high rating category that ranged from 35 degrees to 45 degrees. The pomegranate mobile application users' were distributed on the three categories according to their responses as shown in Table (3). It was found that $66.1 \%$ concentrated in the medium rating category, while the high rating category, represented only $30 \%$ of the total respondents, while the proportion of respondents in the low rating category did not exceed $4 \%$ of the total respondents. Relating to the respondents of the olive mobile applications as shown in Table (4), it was found that $77.8 \%$ concentrated in the medium rating category, and the high rating category represented only $20.5 \%$ of the total respondents, while the proportion of respondents in the low rating category did not exceed $1.7 \%$ of the total respondents.

\subsection{The opinion of respondents regarding the quality of the agricultural information loaded on the applications:}

The results in Table (1) show that the theoretical range for this variable ranged from a minimum of 9 degrees to a maximum of 27 degrees, while the actual range falls between a minimum of 10 degrees to a maximum of 21degrees for the ratings of the pomegranate mobile application users, with a mean of 17.01degrees and a standard deviation of 3.41. While, the results in Table (2) Regarding to the Olive mobile application as mentioned in the show that the actual range was falls between a minimum of 12 degrees to a maximum of 27 degrees, for the ratings of the Olive mobile application users, with a mean of 21.37 degrees and a standard deviation of 3.78.

The theoretical range of the measures of the studied variable was used to construct three categories, 1) a low rating that ranged from 9 degrees to less than 15 degrees, 2) a medium 
Table (1) Descriptive statistics analysis of the users' opinions on the mobile application for Pomegranate

\begin{tabular}{|c|c|c|c|c|c|c|}
\hline \multirow{2}{*}{$\begin{array}{c}\text { Features of the Mobile } \\
\text { Application }\end{array}$} & \multicolumn{2}{|c|}{ theoretical range } & \multicolumn{2}{|c|}{ actual range } & \multirow[b]{2}{*}{ Mean } & \multirow{2}{*}{$\begin{array}{c}\text { Std. } \\
\text { Deviation }\end{array}$} \\
\hline & Minimum & Maximum & Minimum & Maximum & & \\
\hline easiness of using & 15 & 45 & 21 & 39 & 31.65 & 3.98 \\
\hline Quality of Information & 9 & 27 & 10 & 21 & 17.01 & 3.41 \\
\hline Quality of Videos \& Photos & 4 & 12 & 5 & 12 & 9.84 & 1.27 \\
\hline Clarity of Recommendation & 2 & 6 & 2 & 6 & 4.03 & 0.79 \\
\hline
\end{tabular}

Source: Results of the statistical analysis of the study data

Table (2) Descriptive statistics analysis of the users' opinions on the mobile application for Olive

\begin{tabular}{|c|c|c|c|c|c|c|}
\hline \multirow{2}{*}{$\begin{array}{c}\text { Features of the Mobile } \\
\text { Application }\end{array}$} & \multicolumn{2}{|c|}{ theoretical range } & \multicolumn{2}{c|}{ actual range } & & \multicolumn{1}{c|}{$\begin{array}{c}\text { Std. } \\
\text { Deviation }\end{array}$} \\
\cline { 2 - 7 } & Minimum & Maximum & Minimum & Maximum & Mean \\
easiness of using & 15 & 45 & 22 & 44 & 31.18 & 3.66 \\
Quality of Information & 9 & 27 & 12 & 27 & 21.37 & 3.78 \\
Quality of Videos \& Photos & 4 & 12 & 4 & 12 & 9.42 & 1.78 \\
Clarity of Recommendation & 2 & 6 & 2 & 6 & 3.61 & 0.97 \\
\hline
\end{tabular}

Source: Results of the statistical analysis of the study data

rating category that ranged from 15 degrees to less than 21 degrees, 3) and a high rating category that ranged from 21 degrees to 27 degrees. The pomegranate mobile applications users' were distributed on the three categories according to their responses as shown in Table (3). It was found that more than half $(50.7 \%)$ of respondents concentrated in the medium rating category, while $27.3 \%$ in high rating category, while $22 \%$ of the total respondents in the low rating category. As for the respondents of the Olive mobile applications, it was found as show in Table(4) that the majority of respondents $(60.3 \%)$ concentrated in the high rating category, while $33.9 \%$ of the total respondents in the medium rating category, while the proportion of respondents in the low rating category did not exceed $5.9 \%$ of the total respondents.

3.3. The opinion of the respondents regarding the quality of the pictures and videos of the applications:

The results in Table (1) show that the theoretical range for this variable ranged from a minimum of 4 degrees to a maximum of 12 degrees, while the actual range falls between a minimum of 5 degrees to a maximum of 12 degrees for the ratings of the Pomegranate mobile application users, with a mean of 9.84 degrees and a standard deviation of 1.27. While, the results in Table (2) regarding the olive mobile application show that the actual range falls between a minimum of 4 degrees to a maximum of 12 degrees, for the ratings of the Olive mobile application users, with a mean of 9.42 degrees and a standard deviation of 1.78 .

The theoretical range of the measures of the studied variable was used to construct three categories, 1) a low rating category that ranged from 4 degrees to less than 7 degrees, 2) a medium rating category that ranged from 7 degrees to less than 10 degrees, 3) and a high rating category that ranged from 10 degrees to 12 degrees, and the respondents of the Pomegranate mobile applications were distributed on the three categories according to their responses as shown in Table (3) It was found that the majority of respondents $(72.2 \%)$ concentrated in the high rating category, while the medium rating category represented $26 \%$ of the total respondents. while the low rating category represented only $1.8 \%$ of the total respondents. Regarding to the respondents of the olive mobile applications It was found as show in Table(4) that the majority of respondents $61.5 \%$ concentrated in the high rating category, while the medium rating category represented $30.5 \%$ of the total respondents, while the proportion of respondents in the low rating category did not exceed $7.9 \%$ of the total sample. 
Table (3) Distribution of the users' responses to the features of the Pomegranate mobile application according to the responses rating categories

\begin{tabular}{|c|c|c|c|c|c|c|}
\hline \multirow{2}{*}{ Features of the Mobile Application } & \multicolumn{5}{|c|}{ Rating Categories } \\
\cline { 2 - 7 } & \multicolumn{2}{|c|}{ Low } & \multicolumn{2}{c|}{ Medium } & \multicolumn{2}{c|}{ High } \\
\cline { 2 - 7 } & & & & $\%$ & \\
easiness of using & Frequency & & Frequency & & Frequency \\
\hline Quality of Information & 9 & 4 & 150 & 66.1 & 68 & 30 \\
Quality of Videos \& Photos & 62 & 27.3 & 115 & 50.7 & 50 & 22 \\
Clarity of Recommendation & 4 & 1.8 & 59 & 26 & 164 & 72.2 \\
\hline
\end{tabular}

Source: Results of the statistical analysis of the study data

Table (4) Distribution of the users' responses to the features of Olive mobile application according to the responses rating categories

\begin{tabular}{|c|c|c|c|c|c|c|}
\hline \multirow{2}{*}{ Features of the Mobile Application } & \multicolumn{6}{|c|}{ Rating Categories } \\
\cline { 2 - 7 } & \multicolumn{2}{|c|}{ Low } & \multicolumn{2}{c|}{ Medium } & \multicolumn{2}{c|}{ High } \\
\cline { 2 - 7 } & & $\%$ & & $\%$ & & $\%$ \\
\hline easiness of using & Frequency & & Frequency & & Frequency & \\
Quality of Information & 14 & 5.9 & 81 & 33.9 & 144 & 60.3 \\
Quality of Videos \& Photos & 19 & 7.9 & 73 & 30.5 & 147 & 61.5 \\
Clarity of Recommendation & 46 & 19.2 & 34 & 14.2 & 159 & 66.5 \\
\hline
\end{tabular}

Source: Results of the statistical analysis of the study data

3.4. The respondents' willingness to recommend the mobile applications to others:

The results in Table (1) show that the theoretical range for this variable ranged from a minimum of 2 degrees to a maximum of 6 degrees, while the actual range falls between a minimum of 2 degrees to a maximum of 6 degrees for the ratings of the Pomegranate mobile application users, with a mean of 4 degrees and a standard deviation of 0.79 . While the results in Table (2) regarding the olive mobile application show that the actual range falls between a minimum of 2 degrees to a maximum of 6 degrees, for the ratings of Olive mobile application users, with a mean of 3.61 degrees and a standard deviation of 0.97 .

The theoretical range of the measures of the studied variable was used to construct three categories, 1) a low rating category that ranged from 2 degrees to less than 3 degrees, 2) a medium rating category that ranged from 3 degrees to less than 4 degrees, 3) and a high rating category 4 degrees. The pomegranate mobile applications users' were distributed on the three categories according to their responses as shown in Table (3). It was found that the majority of respondents $(82.8 \%)$ concentrated in the high rating category, while the medium rating category, represented only $14.1 \%$ of the total respondents, while the proportion of respondents in the low rating category did not exceed $3.1 \%$ of the total respondents. Regarding the respondents of the Olive mobile applications it was found as show in Table (4) that the majority of respondents $66.5 \%$ concentrated in the high rating category, while the medium rating category, represented $14.2 \%$ of the total respondents, while the low rating category, represented $19.2 \%$ of the total respondents.

3.5. The users' suggestions on other crops that need to develop mobile applications for them:

The results in Table (5) show that the Watermelons was selected by $27.31 \%$ of respondent's pomegranate $\mathrm{M}$. App for mobile application and $0.84 \%$ of respondent's Olive M. App. mango was selected by $27.31 \%$ of respondent's pomegranate M. App and $18.41 \%$ of respondent's Olive M. App. $23.79 \%$ of respondent's pomegranate M. App selected Guava and $22.03 \%$ of them selected Strawberries and Cucumbers. This result indicates to the needs of more Agri.M.app. 
Table (5) Distribution of the responses of mobile application according to the need for mobile applications for more crops

\begin{tabular}{|c|c|c|c|c|}
\hline $\begin{array}{c}\text { Suggested for } \\
\text { mobile } \\
\text { applications }\end{array}$ & $\begin{array}{c}\text { Responses Pomegranate mobile app. } \\
\text { regarding the need for mobile } \\
\text { applications for more crops }\end{array}$ & $\begin{array}{c}\text { Responses Olive mobile app. regarding } \\
\text { the need for mobile applications for } \\
\text { more crops }\end{array}$ \\
\cline { 2 - 5 } & Frequency & $\%$ & Frequency & $\%$ \\
\hline Watermelon & $\mathbf{6 2}$ & $\mathbf{2 7 . 3 1}$ & $\mathbf{2}$ & $\mathbf{0 . 8 4}$ \\
\hline Mango & $\mathbf{6 2}$ & $\mathbf{2 7 . 3 1}$ & $\mathbf{4 4}$ & $\mathbf{1 8 . 4 1}$ \\
\hline Guava & $\mathbf{5 4}$ & $\mathbf{2 3 . 7 9}$ & $\mathbf{0}$ & $\mathbf{0}$ \\
\hline Strawberries & $\mathbf{5 0}$ & $\mathbf{2 2 . 0 3}$ & $\mathbf{0}$ & $\mathbf{0}$ \\
\hline Cucumber & $\mathbf{5 0}$ & $\mathbf{2 2 . 0 3}$ & $\mathbf{0}$ & $\mathbf{0}$ \\
\hline
\end{tabular}

Source: Results of the statistical analysis of the study data

\section{Conclusion}

Smart phones have been identified as one of those effective innovations which benefited a large number of people in the developing world. In Egypt, mobile applications are transforming agriculture to the digital frames and new technology added to farmers. It can be concluded that there were many advantages of the agricultural mobile applications, the most important of them were mentioned as: research results and agricultural recommendations will be communicated to farmers easily and overcome remote distances. Social protection procedures due to the continuity of the current situation regarding the COVID-19 virus (Coronavirus) outbreak can be achieved. The problem of the insufficient number of change agents can be faced.

This research was an attempt to recognize the feedback of the users' of the agricultural applications in order to optimize the benefits of these applications. The assessment would cover identifying the perceptions of the users with regards to their experience with using the applications for Pomegranate and Olive as Eextension services.

The main findings of the research can be summarized as follow: regarding the opinion of the respondents on the quality of the pictures and videos of the applications, it was found that the majority of the Pomegranate application users' $72.2 \%$ are concentrated in the high rating category, against $61.5 \%$ of the Olive application users'. Relating to the respondents' willingness to recommend the mobile applications to others, it was found that the majority of the Pomegranate application users' $82.8 \%$ are concentrated in the high rating category, against $66.5 \%$ of the Olive application users'. Also, according to the questionnaire, Watermelon, then Mango and Guava were selected by respondents to apply within E-Extension. These results indicate that the mentioned applications were useful for their users. This means that these technologies needs enhancement from all of the stockholders either farmers or government or NGOS or research centers or universities.

\section{Acknowledgements}

This work would not have been possible without the financial support of the Agricultural Development Program (ADP), Ministry of Agriculture and Land Reclamation. The authors also gratefully acknowledge the support received from the Central Laboratory for Agricultural Climate (CLAC), Agriculture Research Center (ARC).

\section{REFERENCES}

Abd El-Magieed M. A. M., Sharshar H. A. and Shabana Rasha M. E. (2016). Farmers' Utilization of Mobile Phones to Get Agricultural Information in Dakahlia Governorate ,. J. Agric. Econom. and Social Sci., Mansoura Univ., Vol.7 (5), May.

Aker J.C. (2010). Dial “A” for Agriculture: Using Information and Communication Technologies for Agricultural Extension in Developing Countries. Economics Department and Fletcher School. Tufts University. Medford. MA., USA. 02155.Jenny.Aker@tufts.edu.

Duflo E., Hanna Rema. and Ryan S. (2007). "Monitoring Works: Getting Teachers to Come to School." BREAD Working Paper No. 103.

E-Marketer (2016). Smartphone Users Worldwide Will Total 1.75 Billion in 2014. Retrieved February 14, 2016, from https://www. emarketer.com/Article/Smartphone-UsersWorldwide-Will-Total-175-Billion2014/1010536. 
Kassem M. H. and Shabana Rasha M. E. (2017). Mobile service of the Ministry of Agriculture-Egypt. Karianet and ESDU.

Krejcie R. and Morgan D. W. (1970). " Determining Sample Size for Research Activities in Educational and Psychological Measurement" Vol. (30), Published By College Station, Durham, North Carolina, USA.

Mohamed A. O., Allam Yousria A., Kamel F. K. and Hassen I. I. (2018). Efficiency of Agricultural Extension in the Application of Integrated Control of Olive Fruit Fly in Some Villages of Ismailia Governorate. Bioscience Research. 15(4):3020-3032. Print ISSN: 0253-1496 / Online ISSN: 0976-0539.
Qiang C. Z., Kuek S.C., Andrew D. and Esselaar S. (2011). Mobile Applications for Agriculture and Rural Development. ICT Sector Unit World Bank. December.

Townsend, R., Lampietti J. and others(2019). FUTURE of FOOD Harnessing Digital Technologies to Improve Food System Outcomes. International Bank for Reconstruction and Development / The World Bank.

Trendov N. M., Varas S. and Zeng M (2019). Digital technologies in agriculture and rural areas - Status report. Food and Agriculture Organization of the United Nations. Rome.

$$
\begin{aligned}
& \text { أراء مستخدمي تطبيقات المحمول الخاصة بالإدارة المتكاملة لمحصولى الرمان والزيتون } \\
& \text { أحمد عوني فرج - *عبد العزيز الطويل - *قإنجي خيري فايد } \\
& \text { الدعمل المركزي للمناخ الزراعي ، *معرد بحوث البساتين ،**معهد بحوث الإرشاد الزراعي والتنمبية الريفية }
\end{aligned}
$$

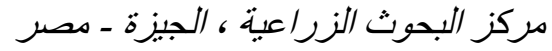

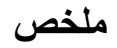

إستهدف البحث التعرف على آراء مستخدمي تطبيق الإدارة المتكامل لمحصولى كل من: الرمان، و الزيتون من

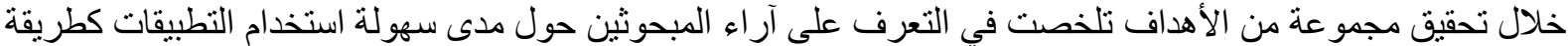

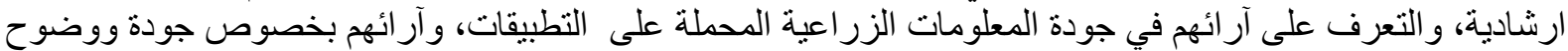

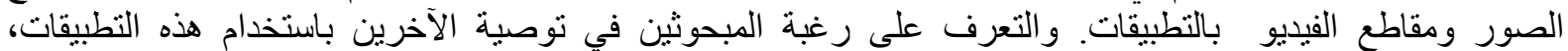

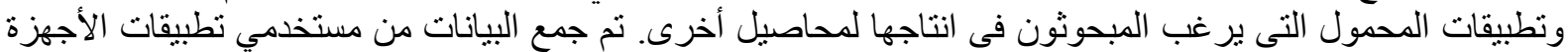

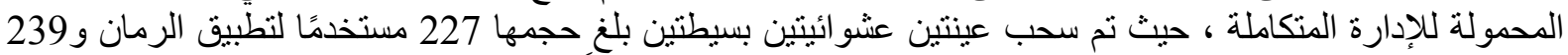

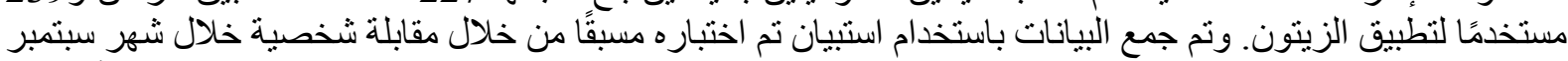

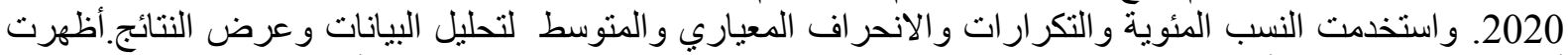

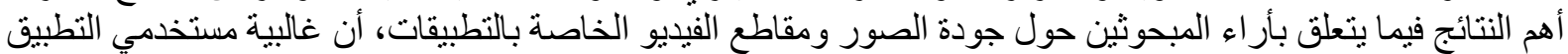

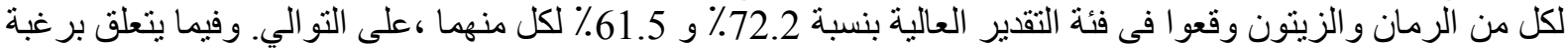
المبحوثين في توصية الآخرين باستخدام تطبيق الهاتف المحمول، فقد وجد أن غالبية مستخدمي التطبيق يتركزون في فئة التئة

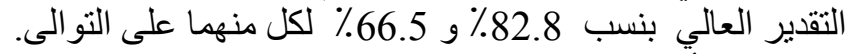

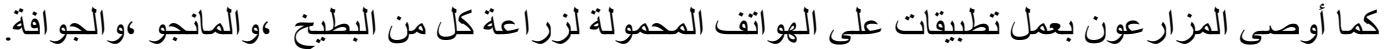
المجلة العلمية لكلية الزراعة - جامعة القاهرة ـ المجلد (71) العدد الرابع (أكتوبر 2020): 243-250 ـ 